\title{
Ultrastructural changes to rabbit fibrin and platelets due to Aspartame
}

\author{
E. Pretorius ${ }^{\text {a }}$; P. Humphries ${ }^{\text {ab }}$ \\ ${ }^{a}$ Department of Anatomy, Faculty of Medicine, University of Pretoria, South Africa \\ ${ }^{b}$ Department of Anatomy, University of Limpopo, Medunsa Campus, Garankuwa, South Africa
}

\begin{abstract}
The coagulation process, including thrombin, fibrin, as well as platelets, plays an important role in hemostasis, contributing to the general well-being of humans. Fibrin formation and platelet activation are delicate processes that are under the control of many small physiological events. Any one of these many processes may be influenced or changed by external factors, including pharmaceutical or nutritional products, e.g., the sweetener aspartame (L-aspartyl-L-phenylalanine methyl ester). It is known that phenylalanine is present at position $\mathrm{P}_{9}$ and aspartate at position $\mathrm{P}_{10}$ of the $\alpha$-chain of human fibrinogen, and plays an important role in the conversion of fibrinogen to fibrin by the catalyst a-thrombin. The authors investigate the effect of aspartame on platelet and fibrin ultrastructure, by using the rabbit animal model and the scanning electron microscope. Animals were exposed to $34 \mathrm{mg} / \mathrm{kg}$ of aspartame $26 \times$ during a 2-month period. Aspartame-exposed fibrin networks appeared denser, with a thick matted fine fiber network covering thick major fibers. Also, the platelet aggregates appeared more granular than the globular control platelet aggregates. The authors conclude by suggesting that aspartame usage may interfere with the coagulation process and might cause delayed fibrin breakup after clot formation. They suggest this, as the fibrin networks from aspartame-exposed rabbits are more complex and dense, due to the netlike appearance of the minor, thin fibers. Aspartame usage should possibly be limited by people on anti-clotting medicine or those with prone to clot formation.
\end{abstract}

\section{Introduction}

The coagulation process, including thrombin, fibrin, as well as platelets, plays an important role in hemostasis, contributing to the general well-being of humans. Thrombin is a serine protease in its highly specific interactions with its primary polypeptide substrate fibrinogen, and is involved in coagulation and fibrinolysis, which are precisely regulated by the measured participation of substrates, activators, inhibitors, cofactors, and receptors. Thrombin is generated by the activation of coagulation, resulting in thrombus formation, when fibrinogen is converted to fibrin after platelet activation. Platelet activation and thrombus formation is therefore dependent on thrombin. The prothrombinase complex is the sole site of thrombin formation in the vasculature. It comprises factor Xa (fXa) assembled with cofactor Va in the presence of calcium $\left(\mathrm{Ca}^{2+}\right)$ on the surface of activated platelets [1]. Thrombin is highly specific in its interaction with fibrinogen, its primary polypeptide substrate, and fibrin formation and platelet activation are therefore delicate processes that are under the control of many small physiological events. Any one of these many processes may, however, be influenced or changed by external factors, including pharmaceutical or nutritional products. One such product is aspartame (L-aspartyl-L-phenylalanine methyl ester). It is known that phenylalanine is present at position $\mathrm{P}_{9}$ and aspartate at position $\mathrm{P}_{10}$ of the A $\alpha$-chain of human fibrinogen, and plays an important role in the conversion of fibrinogen to fibrin by the catalyst a -thrombin [2-4]. Sceheffler and Berliner in 2004 tested the effects of aspartame and its derivatives on human thrombin catalytic activity [5]. They found that the nature of the di-peptide sequence of aspartate as an anionic group at position $\mathrm{P}_{10}$ of the A $\alpha$-chain of human fibrinogen and phenylalanine as an apolar group at position $\mathrm{P}_{9}$, has a remarkable resemblance to adenine nucleotides, e.g., ATP, which are potent inhibitors of thrombin [6,7]. Thus, as aspartame has both phenylalanine and aspartate as key constituents, it could possibly have inhibitory effects on thrombin and so may interfere with the coagulation system. The results reported by Scheffler and Berliner (2004) suggest that fibrinogen binding may be the contributory result of the direct interaction of aspartate-phenylalanine with thrombin [5]. 
However, Sheffler and Berliner in 2004 tried to dismiss any concerns about the effect of aspartame on hemostasis, and the authors suggested that aspartame inhibition of $\alpha$-thrombin probably has little physiological consequence, since the levels required far exceed that ingested, even in overdose amounts [5]. Also, they noted that aspartame is immediately hydrolyzed in the blood by esterases [8,9] and that free aspartate inhibition of fibrinogen clotting would be much weaker than that of aspartame itself, which has an $I_{50}$ of $9 \mathrm{mM}$.

The question that now arises is, does aspartame indeed interfere with platelet activation and fibrin formation or is there no need for concern? Here we investigate whether aspartame affects the coagulation system, by comparing the ultrastructure of platelets and fibrin networks of rabbits expose to $34 \mathrm{mg} / \mathrm{kg}$ [10] of aspartame $26 \times$ during a 2-month period and control rabbit fibrin and platelets. Fibrin clots was prepared from platelet-rich rabbit plasma and human thrombin $[11,12]$.

\section{Materials And Methods}

\section{Study Population: Rabbits}

Ten adult New Zealand white rabbits ( 2 male and 8 female, $\pm 4 \mathrm{~kg}$ ), obtained from a breeding farm in Witbank, Mphumalanga (South Africa), were used for testing aspartame. Ethical clearance was obtained from the University of Pretoria Animal Ethics Committee. Animals were exposed to $34 \mathrm{mg} / \mathrm{kg}$ of aspartame $26 \times$ during a 2-month period. Aspartame-treated rabbits were be fed aspartame by means of force-feeding with a gastric tube. The concentrations of aspartame were determined from the literature and were calculated according to the projected daily ingestion by humans ( $34 \mathrm{mg} / \mathrm{kg}$ body weight) [10]. According to the literature, abuse range is between 100, 150, and $200 \mathrm{mg} / \mathrm{kg}$ body weight [10]. Rabbits in the control group received only double-distilled water by means of force-feeding with a gastric tube. Both the control and test groups received the same volume of fluid, $5 \mathrm{~mL}$. The aspartame-treated rabbits were fed aspartame once in $24 \mathrm{~h}$ for the different exposure period intervals (see Table 1).

Table 1 Different Time Intervals of Aspartame Administration (conc. $34 \mathrm{mg} / \mathrm{kg}$ )

\section{Total number of doses per time}

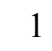

2

7

14

21

28

\section{Different time intervals}

$24 \mathrm{~h}$
$48 \mathrm{~h}$
1 week
2 weeks
3 weeks
4 weeks

\section{Obtaining the Blood Samples}

An average of $1 \mathrm{~mL}$ blood was drawn from the marginal ear vein of the rabbits (without sedation) at the biology section of the production unit (University of Limpopo, Medunsa campus).

\section{Preparation of the Blood Sample to Obtain a Fibrin Clot}

Freshly prepared rabbit platelet-rich plasma (FPRP, from the control and aspartame-treated rabbits utilized in the study) was prepared by drawing $1 \mathrm{~mL}$ of blood, which was centrifuged at $1000 \mathrm{rpm}$ for $2 \mathrm{~min}$. Then $20 \mu \mathrm{L}$ of the FPRP was mixed with $20 \mu \mathrm{L}$ thrombin on a $0.2-\mu \mathrm{m}$ Millipore membrane to form the coagulate (fibrin clot). The filter was placed in a petri dish on filter paper dampened with phosphatebuffered saline (PBS) to create a humid environment and placed a $37^{\circ} \mathrm{C}$ for $10 \mathrm{~min}$. This was followed by a washing process where the millipore membranes containing coagulates were placed in PBS and magnetically stirred for $2 \mathrm{~h}$. Blood proteins trapped within the fibrin network were removed in this way. 


\section{Preparation of the Washed Fibrin Clot for Scanning Electron Microscopy (SEM)}

Washed fibrin clots were fixed in 2.5\% gluteraldehyde in Dulbecco's phosphate-buffered saline (DPBS) buffer with a $\mathrm{pH}$ of 7.4 for $1 \mathrm{~h}$. Each fibrin clot was rinsed 3 times in phosphate buffer for 5 min before being fixed for $1 \mathrm{~h}$ with $1 \%$ Osmium tetra-oxide $\left(\mathrm{OsO}_{4}\right)$. The samples were rinsed 3 times with distilled water for 5 min each rinse and dehydrated serially in 30,50, 70, and 90\% ethanol and 3 times with $100 \%$ ethanol. The SEM procedure was completed by critical point drying of the material, mounting, and examining of the tissue with a JEOL 6000F FEGSEM.

\section{Results And Discussion}

Figure $1 \mathrm{~A}$ and $\mathrm{B}$ shows fibrin fibers and platelets from control rabbits. Fibrin networks contain both major (thick) fibers and minor (thin) fibers (very similar to those of humans) [11, 12]. Two types of fibers are present: major, thick fibers and minor finer fibers. The major fibers are most prominent, with the minor fibers much thinner and appearing between the major, thicker fibers (Figure 1A labels A and B). Rabbit major fiber thickness varies from 0.16 to $0.33 \mu \mathrm{m}$ (Figure 1A). Because platelet-rich plasma was used for all 3 types of clots, platelets were still present trapped between fibrin fibers, or lying on top of the network, to show platelet aggregates. These aggregates are compact and round to oval and have rounded, globular pseudopodia-like projections with the membranes of the aggregates having a smooth membrane surface (Figure 1B, label C).

Figure 1C and D shows fibrin networks and a platelet aggregate from blood exposed to aspartame. Major fibers are present, but the minor, thin fibers form a thick mat over the thicker fibers (label D). These fibers are much more prominent and denser than in the controls. Also, the thin fibers seem to have star-shaped areas from where the fine net project over the thick fibers. Platelets aggregates also differ from control aggregates as they do not have a rounded, globular appearance but are spread out with smaller round areas (label E), forming the body of the aggregate with no typical pseudopodia formation. The aggregate mass also seems less well attached and less compact that that of the controls.

Because of the denseness of the aspartame-exposed fibrin, we suggest that, after clot formation in the body due to small bruises, or minor bleeding events such as bleeding under the skin, the resulting clot will probably take longer to disintegrate. This might be due to the possibility that hemolysis would take longer due to the clot ultrastructure. Platelet structure is also less compact and globular than in the aspartameexposed rabbits. This may additionally affect the coagulation process, but physiological studies are needed to determine the exact mechanism of interaction. 

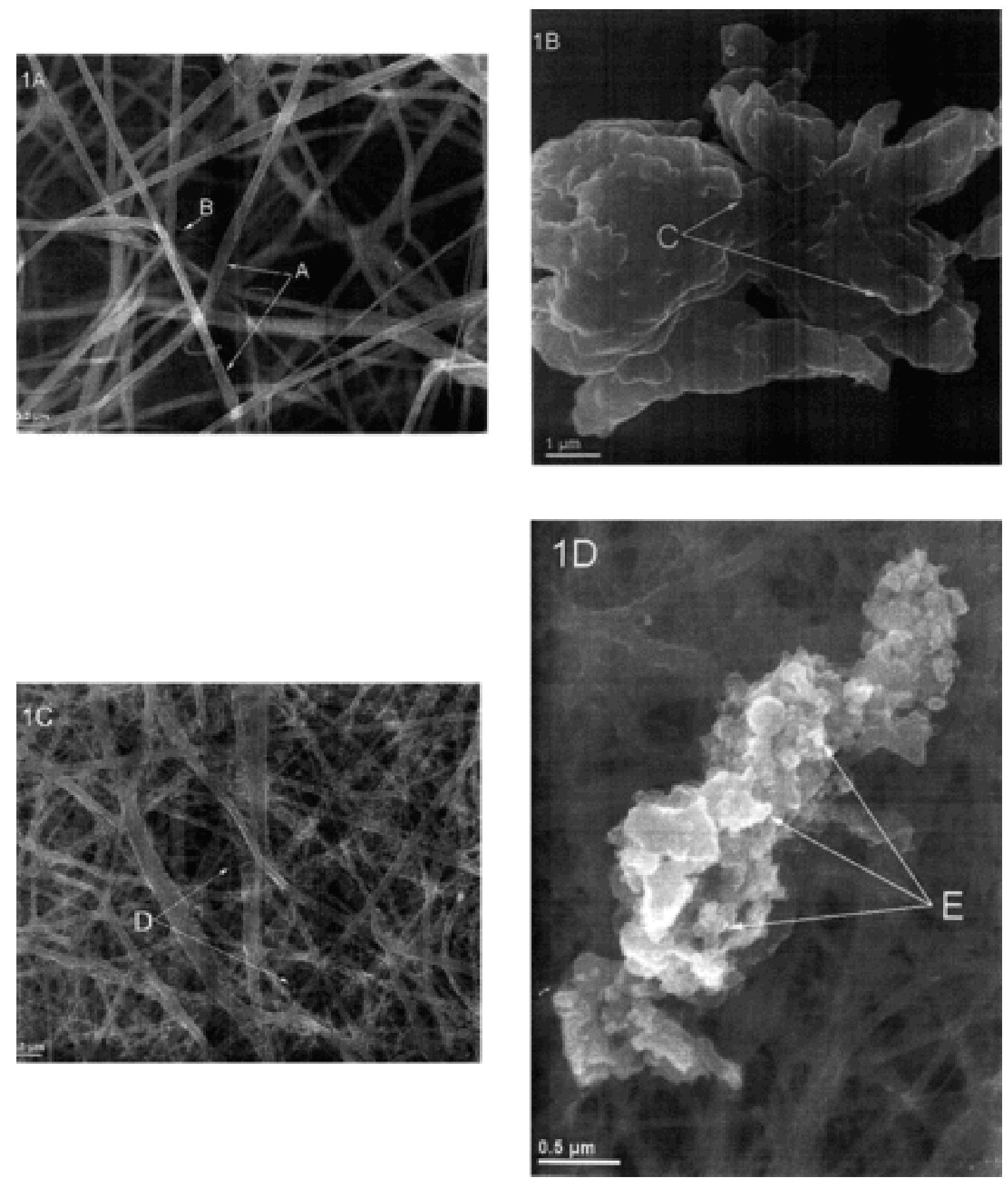

Figure 1. (A) Fibrin network of control rabbit blood rabbit blood clots. Label $\mathrm{A}=$ major fibers; label $\mathrm{B}=$ minor fibers. (B) Platelet aggregate of control rabbit blood. Label $\mathrm{C}=$ pseudopodia. (C) Fibrin network of aspartame-exposed rabbit blood. Label $\mathrm{D}=$ thick minor fiber network. (D) Platelet aggregate of aspartame-exposed rabbit blood. Label $\mathrm{E}=$ small, round, globular areas.

\section{Conclusion}

We conclude by suggesting that aspartame usage may interfere with the coagulation process and might cause delayed fibrin breakup after clot formation. We suggest this, as the fibrin networks from aspartameexposed rabbits are more complex and dense, due to the netlike appearance of the minor, thin fibers. Aspartame usage should therefore possibly be limited by people using anti-clotting medicine and those with prone to clot formation. 


\section{References}

1. Mann, KG, Nesheim, ME, Church, WR, Haley, PE and Krishnaswamy, S. (1990) Surface dependant reactions of the vitamin K-dependant enzyme complexes. Blood 76 , pp. 1-16.

2. Meinwald, YC, Martinelli, RA, van Nispen, JW and Scheraga, HA (1980) Mechanism of action of thrombin on fibrinogen: size of the a alpha fibrinogen-like peptide that contacts the active site of thrombin. Biochemistry 19 , pp. 3820-3825.

3. Marsh Jr, HC, Meinwald, YC, Lee, S. and Scheraga, HA (1982) Mechanism of action of thrombin on fibrinogen: direct evidence for the involvement of pehylalanine at position P9. Biochemistry 24 , pp. 61676171.

4. Marsh Jr, HC, Meinwald, YC, Thannhauser, TW and Scheraga, HA (1983) Mechanism of action of thrombin on fibrinogea: kinetic evidence for involvement of aspartic acid at position P10. Biochemistry 22 , pp. 4170-4174.

5. Scheffler, JE and Berliner, LJ (2004) 2004. Aspartame and aspartame derivatives effect human thrombin catalytic activity. Biophys Chem 112, p. 4170.

6. Conery, BG and Berliner, LJ (1983) Binding subsites in human thrombins. Biochemistry 22 , pp. 369-375.]

7. Berliner, LJ, Birktoft, JJ and Miller, TL (1986) Thrombin: active site topography. Ann N Y Acad Sci. 485 , pp. 80-95.

8. Stegink, LD, Brummel, MC, Filer, LJ and Baker, GL (1983) Blood methanol concentrations in one-year-old infants administered graded doses of aspartame. J Nutr. 113 , pp. 1600-1606.

9. Filer, LJ, Baker, GL and Stegink, LD (1993) Effect of aspartame loading on plasma and erythrocyte free amino acid concentrations in one-year-old infants. J Nutr. 113 , pp. 1591-1599.

10. Stegink, LD, Filer, LJ and Baker, GL (1981) Plasma and erythrocyte concentrations of free amino acids in adult humans administered abuse doses of aspartame. J Toxicol Environ Health 7, pp. 291-305.

11. Pretorius, E., Briedenhann, S., Marx, J. and Franz, RC (2006) Structural changes in the fibrin network of a Pretoria family with dysfibrinogenaemia: a scanning electron microscopical study. Ultrastructural Pathol. 30, pp. 167-176.

12. Pretorius, E., Briedenhann, S., Marx, J., Smit, E., van der Merwe, CF, Pieters, M. and Franz, RC (2007) Ultra-structural comparison of the morphology of three different platelet and fibrin fiber preparations. Anat Rec. 290 , pp. 188-198. 